TITLE:

\title{
Kauffman Bracket Skein Module of the Connected Sum of Handlebodies and Non-injectivity
}

AUTHOR(S):

KARUO, Hiroaki

CITATION:

KARUO, Hiroaki. Kauffman Bracket Skein Module of the Connected Sum of Handlebodies and Non-injectivity. 2021: 1-7: RIMS-1936.

ISSUE DATE:

2021-01

URL:

http://hdl.handle.net/2433/261834

RIGHT: 
RIMS-1936

Kauffman Bracket Skein Module of the Connected Sum of Handlebodies and Non-injectivity

By

Hiroaki KARUO

January 2021

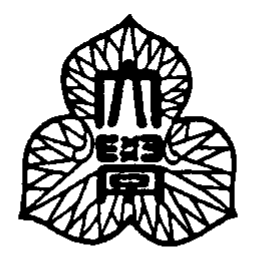

京都大学 数理解析研究所

RESEARCH INSTITUTE FOR MATHEMATICAL SCIENCES

KYOTO UNIVERSITY, Kyoto, Japan 


\title{
KAUFFMAN BRACKET SKEIN MODULE OF THE CONNECTED SUM OF HANDLEBODIES AND NON-INJECTIVITY
}

\author{
HIROAKI KARUO
}

\begin{abstract}
For the handlebody $H_{g}$ of genus $g$, Przytycki studied the (Kauffman bracket) skein module $\mathscr{S}_{q}\left(H_{n} \# H_{m}\right)$ of the connected sum $H_{n} \# H_{m}$ at $q$. One of his results is that, in the case when $1-q^{k}$ is invertible for any $k \neq 0$, a homomorphism $\varphi: \mathscr{S}_{q}\left(H_{n} \sqcup H_{m}\right) \rightarrow \mathscr{S}_{q}\left(H_{n} \# H_{m}\right)$ is an isomorphism, which is induced by a natural way. In this paper, in the case when $n=m=1$, the ground ring is $\mathbb{C}$, and $q \in \mathbb{C}$ is a $4 k$-th root of unity $(k \geq 2)$, we show that $\varphi$ is not injective.
\end{abstract}

\section{INTRODUCTION}

1.1. Skein module of the connected sum of two handlebodies. Let $\mathcal{R}$ be a commutative ring with an identity and a distinguished invertible element $q$, and $M$ be an oriented 3-manifold. The (Kauffman bracket) skein module $\mathscr{S}_{q}(M)$ of $M$ at $q$, introduced by Przytycki [Pr91] and Turaev [Tu91] independently, is the $\mathcal{R}$-module spanned by all isotopy classes of framed unoriented links in $M$ subject to the following two relations (1) and (2), where, in each relation, the framed links are identical except where shown.

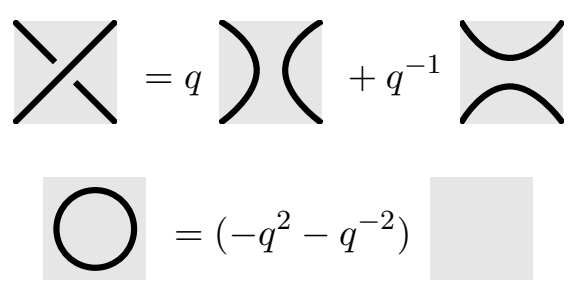

The skein modules have been studied for various kinds of 3-manifolds $M$, for example, the case when $M$ is the connected sum of two 3-manifolds, see e.g. [Pr00], [BP20], [Zh04], [M11], [Pr99]. In this paper, we consider this case.

Let $H_{g}$ denote the handlebody of genus $g$. For two handlebodies $H_{n}$ and $H_{m}$, the connected sum $H_{n} \# H_{m}$ is homeomorphic to the 3-manifold obtained from $H_{g}$ by adding a 2-handle along the curve $\partial D$, where $D$ is a properly embedded disk in $H_{g}$ such that $H_{g} \backslash N(D)$ is homeomorphic to $H_{n} \sqcup H_{m}$, and $N(D)$ is a small open neighborhood of $D$ in $H_{g}$. By regarding isotopy classes of framed oriented links in $H_{n} \sqcup H_{m}$ as those in $H_{g}$, we have a natural $\mathcal{R}$-module homomorphism

$$
\varphi: \mathscr{S}_{q}\left(H_{n}\right) \otimes \mathscr{S}_{q}\left(H_{m}\right) \rightarrow \mathscr{S}_{q}\left(H_{n} \# H_{m}\right) .
$$

In the case when $1-q^{k}$ is invertible in $\mathcal{R}$ for any $k \in \mathbb{Z} \backslash\{0\}$, Przytycki [Pr00] showed that $\varphi$ is an isomorphism.

In the paper, we will consider the case when $\mathcal{R}=\mathbb{C}$ and $q$ is a specified invertible element of $\mathbb{C}^{*}=\mathbb{C} \backslash\{0\}$. Let $\operatorname{ord}(q)$ be the order of $q$, i.e. $q^{\text {ord }(q)}=1$ and $q^{k} \neq 1$ for any $0<k<\operatorname{ord}(q)$. Then, we have the following theorem.

Theorem 1.1. Let $H_{1}$ be the handlebody of genus 1 and $H_{1} \# H_{1}$ be the connected sum of two $H_{1}$ 's. Suppose ord $\left(q^{4}\right) \in \mathbb{Z}_{\geq 2}$. Then, the natural $\mathbb{C}$-module homomorphism

$$
\varphi: \mathscr{S}_{q}\left(H_{1}\right) \otimes \mathscr{S}_{q}\left(H_{1}\right) \rightarrow \mathscr{S}_{q}\left(H_{1} \# H_{1}\right)
$$

is not injective. 
The theorem is proved in Section 3. Note that the case of ord $\left(q^{4}\right)=2$ has been proven in [CT20].

1.2. Organization of the paper. In Section 2, we review skein modules and the skein module of the connected sum of two handlebodies, especially $H_{1} \# H_{1}$. In Section 3, we prove Theorem 1.1 by using the highest degree term with respect to a certain degree.

1.3. Acknowledgments. The author would like to thank Thang T. Q. Le for helpful comments and sharing the result of [CT20]. The author is supported by JSPS KAKENHI Grant Number JP20J10108.

\section{Preliminaries}

Throughout the paper, let $\mathbb{Z}$ be the set of integers, $\mathbb{N}$ be the set of non-negative integers, $\mathbb{C}$ be the set of complex numbers. Let $\Sigma_{g, b}$ denote the connected oriented compact surface with genus $g$ and $b$ boundary components.

2.1. Skein modules. Let $M$ be an oriented 3-manifold. A link in $M$ is a closed unoriented 1dimensional submanifold of $M$. A link $L$ is framed if $L$ is equipped with a framing, i.e. continuous choice of a vector transverse to $L$ at each point of $L$. Let $\mathcal{L}_{f r}$ be the set of all isotopy classes of framed links (including the empty set $\emptyset$ ) in $M$, where two framed links are isotopic if they are isotopic in the class of framed links.

Let $q$ be a specified element in $\mathbb{C}^{*}=\mathbb{C} \backslash\{0\}$. The (Kauffman bracket) skein module $\mathscr{S}_{q}(M)$ of $M$ at $q$ is the $\mathbb{C}$-module spanned by $\mathcal{L}_{f r}$ subject to the skein relation (1) and the trivial loop relation (2), where, in each relation, the framed links are identical except where shown.

For an oriented surface $\Sigma$, in the case of $M=\Sigma \times[0,1]$, we will consider vertically framed links in $M$ in general position as diagrams on $\Sigma$ with respect to the natural projection $\Sigma \times[0,1] \rightarrow$ $\Sigma \times\{0\}=\Sigma$, where a framing of a framed link $L$ is vertical if the vector at each point of $L$ is parallel to $[0,1]$-factor and points to the direction of 1.

It is known that, for the diagrams of two isotopic framed links, one can be obtained from the other by a finite sequence of isotopy of $\Sigma$ and (framed) Reidemeister moves I, II, III depicted in Figure 1, see, for example, [Oh02].
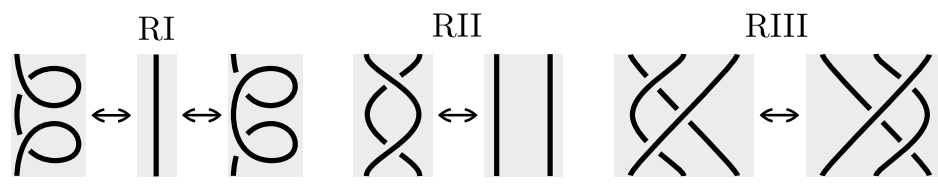

FiguRe 1

For an oriented surface $\Sigma$, if $M=\Sigma \times[0,1]$, then $\mathscr{S}_{q}(M)$ has an algebraic structure by stacking, i.e. for $\alpha_{1}, \alpha_{2} \in \mathcal{L}_{f r}, \alpha_{1} \alpha_{2}$ is defined by stacking: by rescaling $\alpha_{1}$ and $\alpha_{2}$ with respect to [0,1]factor, $\alpha_{1}$ is in $\Sigma \times\left[\frac{1}{2}, 1\right]$ and $\alpha_{2}$ is in $\Sigma \times\left[0, \frac{1}{2}\right]$. In the following, we will write $\mathscr{S}_{q}(\Sigma)$ instead of the skein module $\mathscr{S}_{q}(M)$ with the above algebraic structure.

By using the relations (1) and (2), the following equalities hold, see [Ka87].

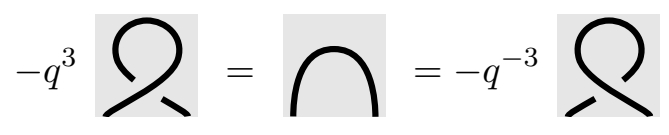


2.2. Connected sum of handlebodies and its skein algebra. Let $H_{g}(g \in \mathbb{N})$ be the handlebody of genus $g$ and let $H_{1} \# H_{1}$ denote the connected sum of two $H_{1}$ 's. Let $D$ be a properly embedded closed disk in $H_{2}$ such that each component of $H_{2} \backslash N(D)$ is homeomorphic to $H_{1}$, where $N(D)$ is a small open neighborhood of $D$ in $H_{2}$. We put $\gamma=\partial D$. Let $\left(H_{m}\right)_{\gamma}$ be the 3-manifold obtained by adding a 2-handle to $H_{m}$ along $\gamma$. Then, note that $H_{1} \# H_{1}=\left(H_{2}\right)_{\gamma}$. Hence, there is a natural inclusion $i: H_{2} \hookrightarrow\left(H_{2}\right)_{\gamma}=H_{1} \# H_{1}$. The natural inclusion $i: H_{2} \hookrightarrow H_{1} \# H_{1}$ induces a $\mathbb{C}$-module homomorphism $i_{*}: \mathscr{S}_{q}\left(H_{2}\right) \rightarrow \mathscr{S}_{q}\left(H_{1} \# H_{1}\right)$.

In the following, we assume $H_{g}=\Sigma_{0, g+1} \times[0,1]$. Let $x_{1}, x_{2}, y$ be the framed links (not isotopy classes) in $H_{2}$ depicted in Figure 2, where $x_{1}, x_{2}, y$ are depicted on $\Sigma_{0,3}$ as diagrams with respect to the natural projection $H_{2}=\Sigma_{0,3} \times[0,1] \rightarrow \Sigma_{0,3}$. It is known that $\mathscr{S}_{q}\left(H_{2}\right)=\mathbb{C}\left[x_{1}, x_{2}, y\right]$ as $\mathbb{C}$-modules, see $[\operatorname{Pr} 91]$.

From $H_{g}=\Sigma_{0, g+1} \times[0,1]$, recall that $\mathscr{S}_{q}\left(H_{g}\right)=\mathscr{S}_{q}\left(\Sigma_{0, g+1}\right)$ has an algebraic structure by stacking. For two framed links $L_{1}, L_{2} \subset H_{2}$, one can consider the product $L_{1} L_{2} \in \mathscr{S}_{q}\left(\Sigma_{0,3}\right)$. In the proofs of lemmas, we will use this notation.
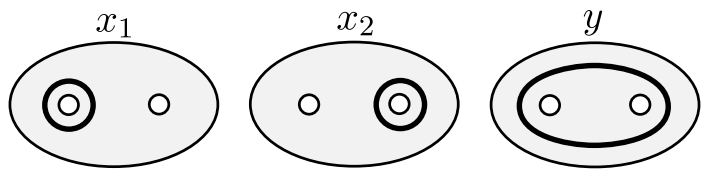

Figure 2

Let $d$ be a properly embedded closed interval in $\Sigma_{0,3}$ such that each component $\Sigma_{0,3} \backslash N(d)$ is homeomorphic to $\Sigma_{0,2}$, where $N(d)$ is a small open tubular neighborhood of $d$ in $\Sigma_{0,3}$. In the following, we assume that the separating disk $D \subset H_{2}=\Sigma_{0,3} \times[0,1]$ is equal to $d \times[0,1]$.

Let $z_{k}\left(k \in \mathbb{Z}_{>1}\right)$ be a framed link (not isotopy class) in $H_{2}$ such that $z_{k}$ intersects transversely with $D \subset H_{2} 2 k$ times. Then, $i_{*}\left(z_{k}\right)=i_{*}\left(q^{6} u\left(z_{k}\right)\right)$ in $\mathscr{S}_{q}\left(H_{1} \# H_{1}\right)$ obtained from a handle sliding relation $i\left(z_{k}\right)=i\left(s l_{\gamma}\left(z_{k}\right)\right)$ in $H_{1} \# H_{1}$ by (positive) handle sliding on the top arc of $z_{k}$ along $\gamma=\partial D$ depicted in Figure 3, where the shaded region is a small neighborhood of $d$ in $\Sigma_{0,3}$.

Let $y^{k}$ be the framed link (not isotopy class) in $H_{2}$ depicted in Figure 4 . In the following, let $u\left(y^{k}\right)$ be denoted by $u_{k}$, see Figure 4 . Then, $i_{*}\left(y^{k}\right)=i_{*}\left(q^{6} u_{k}\right)$ in $\mathscr{S}_{q}\left(H_{1} \# H_{1}\right)$.

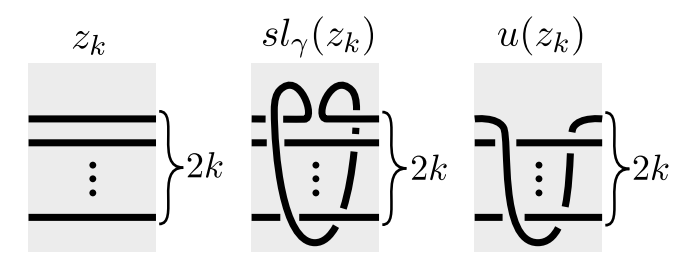

Figure 3

Let $\mathcal{L}_{f r}^{g e n}$ be a set of framed links (not isotopy classes) in $H_{2}$ generating $\mathscr{S}_{q}\left(H_{2}\right)$ such that each $L \in \mathcal{L}_{f r}^{g e n}$ intersects with $D$ transversely. Then, it is known that $\mathscr{S}_{q}\left(H_{1} \# H_{1}\right)=\mathscr{S}_{q}\left(H_{2}\right) / K$, where $K$ is the $\mathbb{C}$-submodule of $\mathscr{S}_{q}\left(H_{2}\right)$ generated by $L-s l_{\gamma}(L)$ for all $L \in \mathcal{L}_{f r}^{g e n}$, and $s l_{\gamma}(L)$ is obtained from $L \in \mathcal{L}_{f r}^{g e n}$ by handle sliding $L$ along $\gamma=\partial D$, see Lemma 4.1 in [Pr00] for more details.

Let $b\left(y^{k}\right)$ denote the framed link (not isotopy class) in $H_{2}$ depicted in Figure 4. 

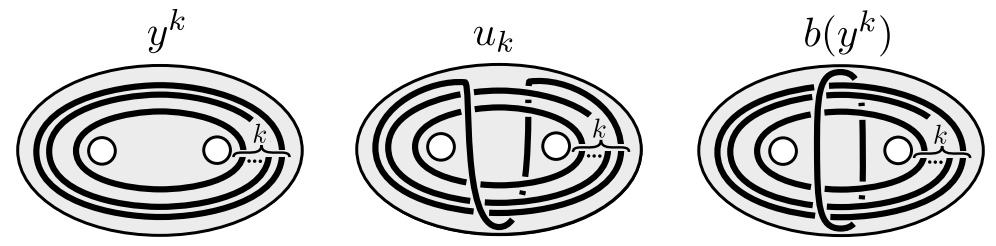

FIGURE 4

Lemma 2.1. For $k \in \mathbb{Z}_{\geq 2}$, in $\mathscr{S}_{q}\left(H_{2}\right)$,

$$
u_{k}=q^{4} y u_{k-1}-q^{-4}\left(1-q^{4}\right) x_{1} x_{2} y^{k-1}-q^{-2}\left(1-q^{4}\right)\left(x_{1}^{2}+x_{2}^{2}\right) y^{k-2}+\left(q^{4}-q^{-4}\right) b\left(y^{k-2}\right) .
$$

Proof. By resolving the bottom two crossings, we have

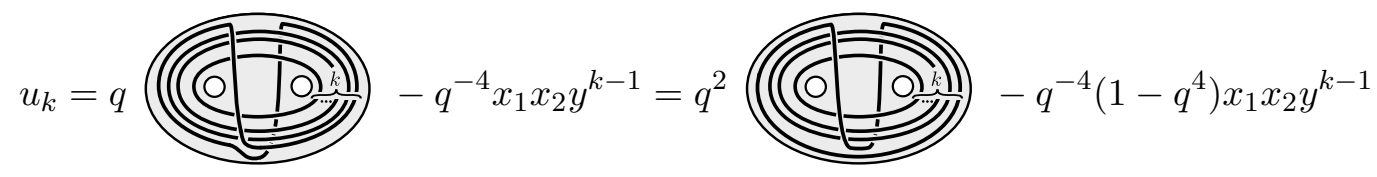

in $\mathscr{S}_{q}\left(H_{2}\right)$, where the first equality follows from (3).

To show the claim, it is enough to show

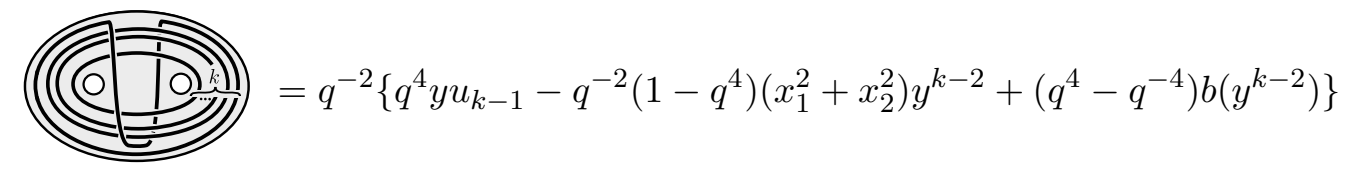

in $\mathscr{S}_{q}\left(H_{2}\right)$. Actually, we have

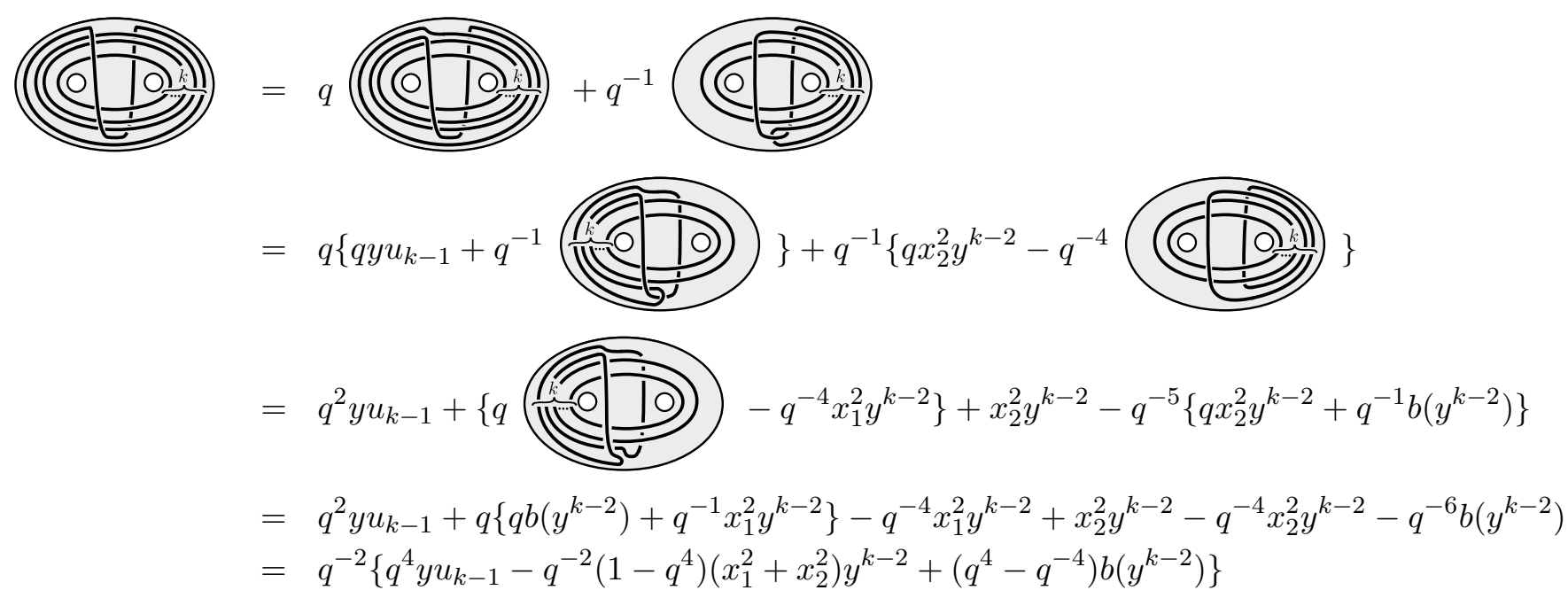

in $\mathscr{S}_{q}\left(H_{2}\right)$, where the first equality follows by applying (1) to the upper left crossing, and the second equality follows by applying (1) to the upper right crossing in the first term and by applying (1) to the bottom crossing in the second term then applying (3), and the third equality follows by applying (1) to the bottom crossing in the second term and the top crossing in the fourth term, and the fourth equality follows by applying (1) to the bottom crossing in the second term. 
KAUFFMAN BRACKET SKEIN MODULE OF THE CONNECTED SUM OF HANDLEBODIES AND NON-INJECTIVITצ

Proposition 2.2. For $k \in \mathbb{Z}_{\geq 2}$, in $\mathscr{S}_{q}\left(H_{2}\right)$,

$u_{k}=q^{4 k-2} y^{k}-q^{-4}\left(1-q^{4 k}\right) x_{1} x_{2} y^{k-1}-q^{-2}\left(1-q^{4(k-1)}\right)\left(x_{1}^{2}+x_{2}^{2}\right) y^{k-2}+\left(q^{4}-q^{-4}\right) \sum_{i=0}^{k-2}\left(q^{4} y\right)^{i} b\left(y^{k-i-2}\right)$.

Proof. In the case of $k=2$, by using only the skein relation (1) and the trivial loop relation (2), we have

$$
u_{2}=q^{4} y u_{1}-q^{-4}\left(1-q^{4}\right) x_{1} x_{2} y-q^{-2}\left(1-q^{4}\right)\left(x_{1}^{2}+x_{2}^{2}\right)+\left(q^{4}-q^{-4}\right)\left(-q^{2}-q^{-2}\right)
$$

in $\mathscr{S}_{q}\left(H_{2}\right)$. Since $b\left(y^{0}\right)=b(\emptyset)=-q^{2}-q^{-2}$ in $\mathscr{S}_{q}\left(H_{2}\right)$, the claim holds for $k=2$.

Assume that the claim holds for $k=j \geq 2$. From Lemma 2.1,

$$
\begin{aligned}
u_{j+1}= & q^{4} y u_{j}-q^{-4}\left(1-q^{4}\right) x_{1} x_{2} y^{j}-q^{-2}\left(1-q^{4}\right)\left(x_{1}^{2}+x_{2}^{2}\right) y^{j-1}+\left(q^{4}-q^{-4}\right) b\left(y^{j-1}\right) \\
= & q^{4} y\left\{q^{4 j-2} y^{j}-q^{-4}\left(1-q^{4 j}\right) x_{1} x_{2} y^{j-1}-q^{-2}\left(1-q^{4(j-1)}\right)\left(x_{1}^{2}+x_{2}^{2}\right) y^{j-2}+\left(q^{4}-q^{-4}\right) \sum_{i=0}^{j-2}\left(q^{4} y\right)^{i} b\left(y^{j-i-2}\right)\right\} \\
& -q^{-4}\left(1-q^{4}\right) x_{1} x_{2} y^{j}-q^{-2}\left(1-q^{4}\right)\left(x_{1}^{2}+x_{2}^{2}\right) y^{j-1}+\left(q^{4}-q^{-4}\right) b\left(y^{j-1}\right) \\
= & q^{4(j+1)-2} y^{j}-q^{-4}\left(1-q^{4(j+1)}\right) x_{1} x_{2} y^{j}-q^{-2}\left(1-q^{4 j}\right)\left(x_{1}^{2}+x_{2}^{2}\right) y^{j-1}+\left(q^{4}-q^{-4}\right) \sum_{i=0}^{j-1}\left(q^{4} y\right)^{i} b\left(y^{j-i-1}\right)
\end{aligned}
$$

in $\mathscr{S}_{q}\left(H_{2}\right)$. Hence, the claim holds for $k=j+1$ holds.

Since $i_{*}\left(y^{k}\right)=i_{*}\left(q^{6} u_{k}\right)$ in $\mathscr{S}_{q}\left(H_{1} \# H_{1}\right)$, from Proposition 2.2 ,

$$
i_{*}\left(\left(1-q^{4 k+4}\right) y^{k}\right)=i_{*}\left(-q^{2}\left(1-q^{4 k}\right) i_{*}\left(x_{1} x_{2} y^{k-1}\right)-q^{4}\left(1-q^{4(k-1)}\right)\left(x_{1}^{2}+x_{2}^{2}\right) y^{k-2}+q^{6}\left(q^{4}-q^{-4}\right) \sum_{i=0}^{k-2}\left(q^{4} y\right)^{i} b\left(y^{k-i-2}\right)\right)
$$

in $\mathscr{S}_{q}\left(H_{1} \# H_{1}\right)$ for any $k \in \mathbb{Z}_{\geq 2}$.

Remark 2.3. By concrete calculation, we have

$$
u_{1}=q^{2} y-q^{-4}\left(1-q^{4}\right) x_{1} x_{2}
$$

in $\mathscr{S}_{q}\left(H_{2}\right)$ : a formula similar to (5) is written in [BP20] in terms of Temperley-Lieb module. In particular, since $i_{*}(y)=i_{*}\left(q^{6} u_{1}\right)$ in $\mathscr{S}_{q}\left(H_{1} \# H_{1}\right)$, we have

$$
i_{*}\left(\left(1-q^{8}\right) y\right)=i_{*}\left(q^{2}\left(1-q^{4}\right) x_{1} x_{2}\right)
$$

in $\mathscr{S}_{q}\left(H_{1} \# H_{1}\right)$.

\section{Non-INJECTIVITY}

In this section, we will prove Theorem 1.1, i.e. if $q \in \mathbb{C}^{*}$ is a $4 k$-th root of unity $\left(k \in \mathbb{Z}_{\geq 2}\right)$, then $\varphi: \mathscr{S}_{q}\left(H_{1}\right) \otimes \mathscr{S}_{q}\left(H_{1}\right) \rightarrow \mathscr{S}_{q}\left(H_{1} \# H_{1}\right)$ is not injective.

3.1. Main result. Note that $\mathscr{S}_{q}\left(H_{1}\right)$ has a basis $\left\{x^{n} \mid n \in \mathbb{N}\right\}$ as a $\mathbb{C}$-module, where $x$ is the framed link (not isotopy class) depicted in Figure 5, where the diagram is depicted on $\Sigma_{0,2}$. Then, there is a natural homomorphism

$$
\varphi: \mathscr{S}_{q}\left(H_{1}\right) \otimes \mathscr{S}_{q}\left(H_{1}\right) \rightarrow \mathscr{S}_{q}\left(H_{1} \# H_{1}\right)
$$

obtained by extending the correspondence $x^{n} \otimes x^{m} \mapsto i_{*}\left(x_{1}^{n} x_{2}^{m}\right)(n, m \in \mathbb{N})$ linearly, where $x_{1}$ and $x_{2}$ are the framed links in $H_{2}$ depicted in Figure 2, and $x_{1}^{n} x_{2}^{m} \in \mathscr{S}_{q}\left(\Sigma_{0,3}\right)$ is defined in subsection 2.1 , and $i_{*}$ is the $\mathbb{C}$-module homomorphism $\mathscr{S}_{q}\left(\Sigma_{0,3}\right) \rightarrow \mathscr{S}_{q}\left(H_{1} \# H_{1}\right)$ induced from the inclusion 
$i: \Sigma_{0,3} \times[0,1]=H_{2} \hookrightarrow\left(H_{2}\right)_{\gamma}=H_{1} \# H_{1}$. It is known that if $q$ is not a root of unity, then $\varphi$ is an isomorphism, see Corollary 6.2 in [Pr00] for more details.

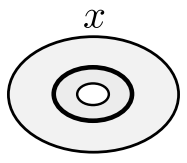

Figure 5

In the following, we will consider the case when $q$ is a root of unity. Recall that $\operatorname{ord}(q)$ is the order of $q$, i.e. $q^{\text {ord }(q)}=1$ and $q^{k} \neq 1$ for any $0<k<\operatorname{ord}(q)$.

Proof of Theorem 1.1. We have a $\mathbb{C}$-module homomorphism $\psi: \mathscr{S}_{q}\left(H_{1}\right) \otimes \mathscr{S}_{q}\left(H_{1}\right) \rightarrow \mathbb{C}\left[x_{1}, x_{2}\right]$ by extending the correspondence $x^{n} \otimes x^{m} \mapsto x_{1}^{n} x_{2}^{m}$ linearly. In particular, $\psi$ is an isomorphism. We consider $\varphi \circ \psi^{-1}: \mathbb{C}\left[x_{1}, x_{2}\right] \rightarrow \mathscr{S}_{q}\left(H_{1} \# H_{1}\right)$. By regarding $\mathbb{C}\left[x_{1}, x_{2}\right]$ as a $\mathbb{C}$-submodule of $\mathbb{C}\left[x_{1}, x_{2}, y\right]=\mathscr{S}_{q}\left(H_{2}\right), \varphi \circ \psi^{-1}$ maps $z \in \mathbb{C}\left[x_{1}, x_{2}\right]$ to $i_{*}(z) \in \mathscr{S}_{q}\left(H_{1} \# H_{1}\right)$. To show the claim, it is enough to show that there is a non-trivial element $z \in \mathbb{C}\left[x_{1}, x_{2}\right]$ such that $i_{*}(z)=0$ in $\mathscr{S}_{q}\left(H_{1} \# H_{1}\right)$.

In the case of ord $\left(q^{4}\right)=2$, from $(6)$,

$$
i_{*}\left(-q^{2}\left(1-q^{4}\right) x_{1} x_{2}\right)=-q^{2}\left(1-q^{4}\right) i_{*}\left(x_{1} x_{2}\right)=0
$$

in $\mathscr{S}_{q}\left(H_{1} \# H_{1}\right)$. Since $-q^{2}\left(1-q^{4}\right) \in \mathbb{C}^{*}, i_{*}\left(x_{1} x_{2}\right)=0$ in $\mathscr{S}_{q}\left(H_{1} \# H_{1}\right)$.

In the following of the proof, we suppose $\operatorname{ord}\left(q^{4}\right)=N \geq 3$.

Define the degree of $x_{1}^{n} x_{2}^{m}$ by $\operatorname{deg}\left(x_{1}^{n_{1}} x_{2}^{n_{2}}\right)=n_{1}+n_{2}$. Then, it induces an $\mathbb{N}$-filtration on $\mathbb{C}\left[x_{1}, x_{2}\right]$.

The equations (4) with $k=1, \ldots, N-2$ show that, for $k \geq N-2$, one has

$$
i_{*}\left(y^{k}\right) \sim i_{*}\left(\left(x_{1} x_{2}\right)^{k}+\text { lower degree terms }\right)
$$

in $\mathscr{S}_{q}\left(H_{1} \# H_{1}\right)$, where $a \sim b$ means $a=\lambda b(0 \neq \lambda \in \mathbb{C})$.

The equation (4) with $k=N-1$ shows, in $\mathscr{S}\left(H_{1} \# H_{1}\right)$,

$$
i_{*}\left(\left(x_{1} x_{2}\right)^{N-1}\right) \sim i_{*}(\text { lower degree terms }) .
$$

We put the lower degree terms by $p\left(x_{1}, x_{2}\right)$. Then, there is $0 \neq \lambda \in \mathbb{C}$ such that

$$
i_{*}\left(\left(x_{1} x_{2}\right)^{N-1}-\lambda p\left(x_{1}, x_{2}\right)\right)=0
$$

in $\mathscr{S}_{q}\left(H_{1} \# H_{1}\right)$. However, the left-hand side of $(7)$ is not zero in $\mathbb{C}\left[x_{1}, x_{2}\right]$ since $\operatorname{deg}\left(\left(x_{1} x_{2}\right)^{N-1}\right)=$ $2 N-2$ and $\operatorname{deg}\left(p\left(x_{1}, x_{2}\right)\right)<2 N-2$.

Remark 3.1. Note that $\mathscr{S}_{q}\left(H_{1} \# H_{1}\right)$ is a $\mathbb{C}$-vector space, which has no torsion elements. Whereas, since if $L \in K$ then $x_{i} L \in K(i=1,2)$, i.e. $x_{i} K \subset K(i=1,2), \mathscr{S}_{q}\left(H_{1} \# H_{1}\right)=\mathscr{S}_{q}\left(H_{2}\right) / K$ has a $\mathbb{C}\left[x_{1}, x_{2}\right]$-module structure. In particular, Theorem 1.1 implies that the empty set $\emptyset$ is a torsion element in $\mathscr{S}_{q}\left(H_{1} \# H_{1}\right)$ as a $\mathbb{C}\left[x_{1}, x_{2}\right]$-module, which is killed by a degree $2 N-2$ polynomial in $\mathbb{C}\left[x_{1}, x_{2}\right]$.

\section{REFERENCES}

[BP20] R. P. Bakshi, J. H. Przytycki, Kauffman Bracket Skein Module of the Connected Sum of Handlebodies: A Counterexample. Preprint, 2020. arXiv:2005.07750 [math.GT].

[CT20] F. Costantino, T. T. Q. Le, TQFT associated with stated skein module of marked 3-manifold. preprint 2020.

[Ka87] L. Kauffman, State models and the Jones polynomial. Topology 26 (1987), no. 3, 395-407. 
[M11] M. Mroczkowski, Kauffman bracket skein module of the connected sum of two projective spaces. J. Knot Theory Ramifications 20 (2011), no. 5, 651-675.

[Oh02] T. Ohtsuki, Quantum invariants. A study of knots, 3-manifolds, and their sets. Series on Knots and Everything, 29. World Scientific Publishing Co., Inc., River Edge, NJ, 2002.

[Pr91] J. H. Przytycki, Skein modules of 3-manifolds. Bull. Polish Acad. Sci. Math. 39 (1991), no. 1-2, 91-100.

[Pr99] J. H. Przytycki, Fundamentals of Kauffman bracket skein modules. Kobe J. Math. 16 (1999), no. 1, 45-66.

[Pr00] J. H. Przytycki, Kauffman bracket skein module of a connected sum of 3-manifolds. Manuscripta Math. 101 (2000), no. 2, 199-207.

[Tu91] V. G. Turaev, Skein quantization of Poisson algebras of loops on surfaces. Ann. Sci. cole Norm. Sup. (4) 24 (1991), no. 6, 635-704.

[Zh04] J. K. Zhong, The Kauffman skein module of a connected sum of 3-manifolds. Topology Appl. 139 (2004), no. $1-3,113-128$

Research Institute for Mathematical Sciences, Kyoto University, Sakyo-ku, Kyoto, 606-8502, JAPAN

E-mail address: karu@kurims.kyoto-u.ac.jp 